\title{
Listeria Rhombencephalitis
}

\author{
Valappil V. Ashraf ${ }^{1}$ Kizhakkaniyath Abdul Salam \\ ${ }^{1}$ Department of Neurology, Meitra Hospital, Calicut, Kerala, India
}

Address for correspondence Ashraf V. Valappil, DM, Department of Neurology, Meitra Hospital, Edakkad PO, Calicut 673004, Kerala, India (e-mail: drvvashraf@hotmail.com).
Rhombencephalitis is the inflammation of brainstem and cerebellum (hindbrain) and has a variety of potential underlying etiologies that respond variably to treatment, including infections, inflammatory disorders, parainfectious disorders, and paraneoplastic syndromes. ${ }^{1}$ Listeria is the most common infectious agent causing rhombencephalitis and mostly affects immunocompromised individuals.

A 48-year-old man with background history of multiple myeloma (on chemotherapy with bortezomib, thalidomide, and dexamethasone) was admitted with right hemiplegia and altered mental status. His symptoms began 10 days ago immediately after last course of chemotherapy, with low-grade fever and headache, followed by right hemiparesis. He was hospitalized at another center with a probable diagnosis of acute disseminated encephalomyelitis (ADEM) or herpes encephalitis and was treated with acyclovir and steroids. Subsequently his neurological status gradually worsened and he became quadriparetic and unresponsive. On arrival to our hospital, he was febrile, unresponsive (Glasgow Coma Scale [GCS] of 4/15). He had right third, fourth, and sixth cranial nerve palsy and asymmetric quadriparesis, right side more severely affected. Magnetic resonance imaging (MRI) brain revealed irregular confluent areas of T2 and fluid-attenuated inversion recovery (FLAIR) hyperintensities involving frontal and temporal white matter, both internal capsule, midbrain, pons, and bilateral middle cerebellar peduncle ( - Fig. 1). Minimal patchy diffusion restriction was noted in bilateral middle cerebellar peduncles and internal capsule (-Fig. 2). Patchy contrast enhancement was noted in dorsal midbrain, pons, and part of right internal capsule ( - Fig. 3). Blood investigations revealed leukocytosis, normal renal function, and liver function tests. Cerebrospinal fluid (CSF) analysis revealed raised protein of $92 \mathrm{mg} / \mathrm{dL}$, normal glucose, and elevated white cell count of 135 cells $/ \mathrm{mm}^{3}$ (L68\% and P32\%). Blood culture and CSF culture grew Listeria monocytogenes confirming the diagnosis of Listeria rhombencephalitis. He was treated with ampicillin injection for 4 weeks and oral cotrimoxazole for 8 weeks, and he recovered well with mild residual quadriparesis.

Encephalitis due to L. monocytogenes shows predilection for the brainstem, cerebellar peduncle, and cerebellum as in this patient. Other infrequent sites of involvement include supratentorial white matter, thalamus, internal capsule, and spinal cord. ${ }^{1,2}$ Most common MRI feature is
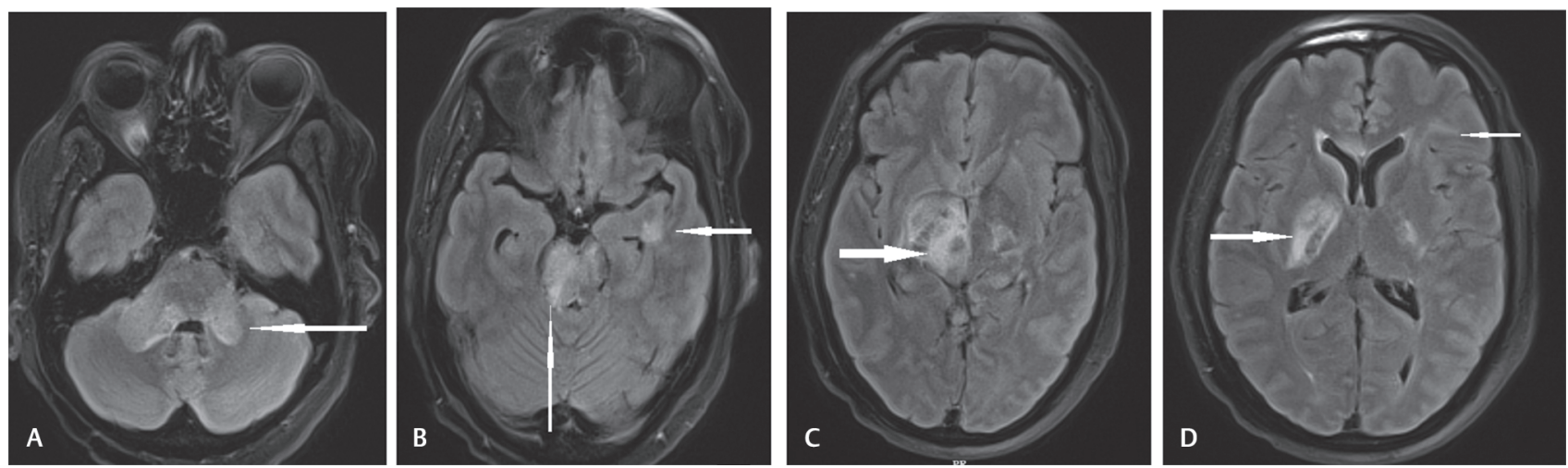

Fig. 1 MRI T2 FLAIR axial sequences showing (A) bilateral symmetrical hyperintensities involving middle cerebellar peduncle(MCP); (B) hyperintensities involving pons and left temporal white matter; (C) hyperintensities involving midbrain; and (D) hyperintensities involving both internal capsule, left frontal white matter, and corpus callosum. FLAIR, fluid-attenuated inversion recovery; MRI, magnetic resonance imaging.

published online

March 24, 2021
DOI https://doi.org/

$10.1055 / \mathrm{s}-0041-1726648$

ISSN 0976-3147
(C2021. Association for Helping Neurosurgical Sick People.

This is an open access article published by Thieme under the terms of the Creative Commons Attribution-NonDerivative-NonCommercial-License, permitting copying and reproduction so long as the original work is given appropriate credit. Contents may not be used for commercial purposes, or adapted, remixed, transformed or built upon. (https://creativecommons.org/licenses/by-nc-nd/4.0/).

Thieme Medical and Scientific Publishers Pvt. Ltd. A-12, 2nd Floor, Sector 2, Noida-201301 UP, India 

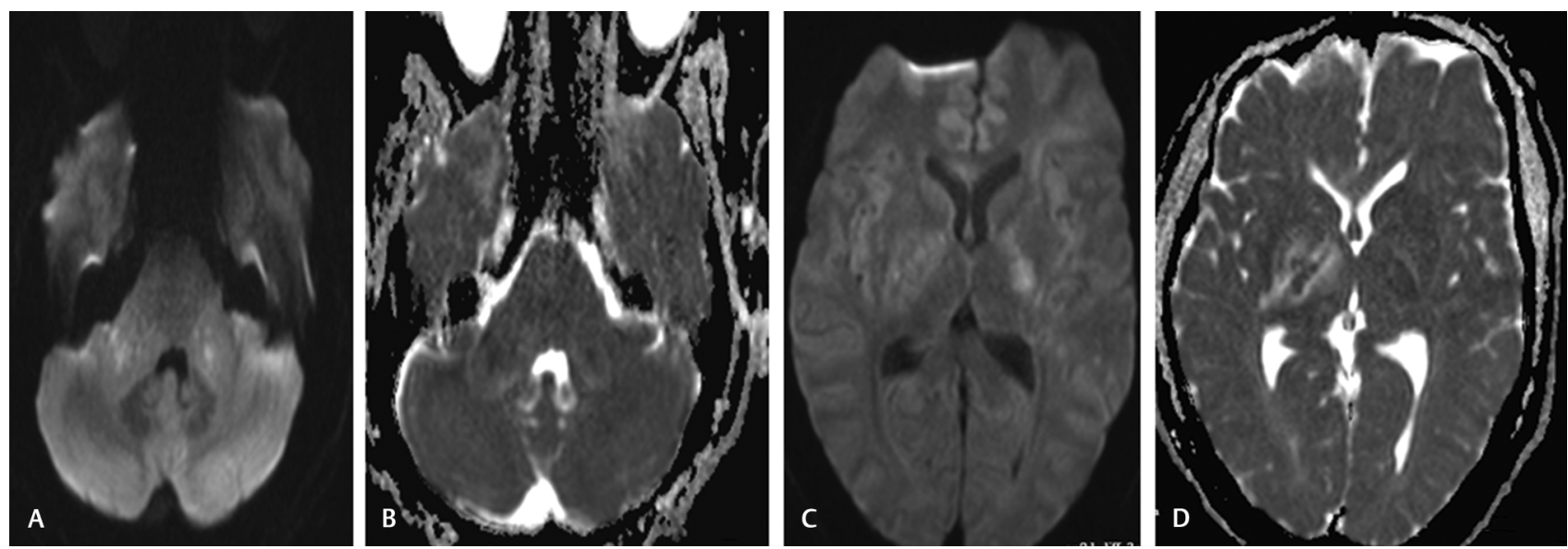

Fig. 2 MRI (A) diffusion weighted imaging (DWI) showing hyperintensities involving both middle cerebellar peduncle(MCP), (B) corresponding apparent diffusion coefficient (ADC) sequence showing hypointensity of MCP suggestive of diffusion restriction, (C) DWI showing hyperintensities involving both internal capsules, and (D) corresponding ADC slowing corresponding hypointensities involving both internal capsule. MRI, magnetic resonance imaging.
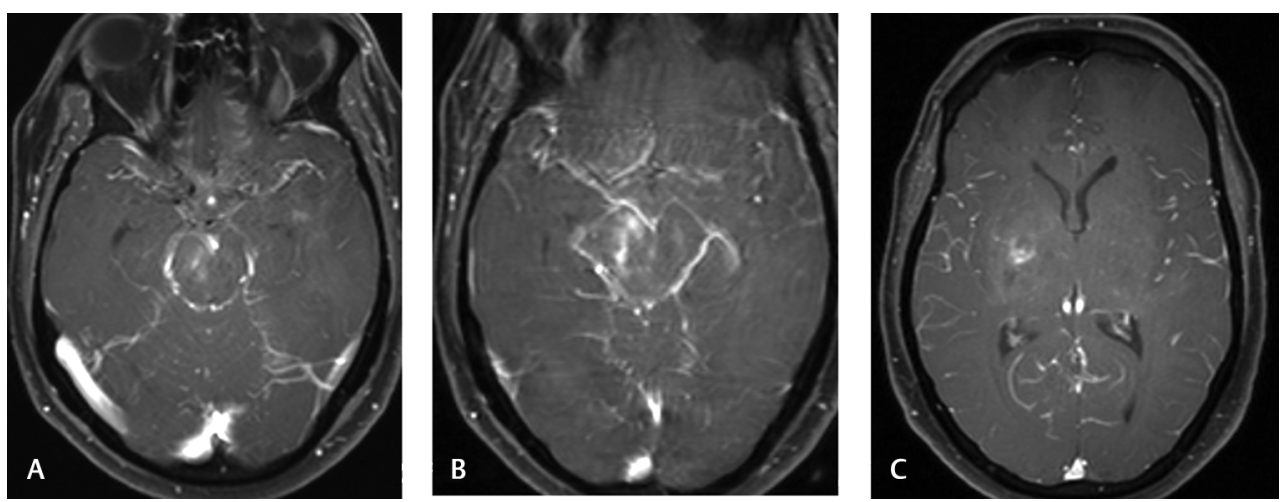

Fig. 3 MRI Postcontrast T1 sequence showing patchy contrast enhancement involving (A) pons (B) midbrain (C) right internal capsule. MRI, magnetic resonance imaging.

the T2 hyperintensity followed by contrast enhancement as seen in this patient. ${ }^{1}$ Other MRI findings include abscess formation, hydrocephalus, edema, and hemorrhages. We noted patchy areas of diffusion restriction indicating areas of necrosis. The regions of restricted diffusion were surrounded by hyperintense signal on $\mathrm{T} 2$-weighted images that most likely denoted as edema. ${ }^{2,3}$ The typical distribution patterns in $L$. rhombencephalitis may be due to the proliferation and spread of L. monocytogenes along the intracerebral tracts. ${ }^{4,5}$ In rhombencephalitis, lesions are usually confined to brainstem and cerebellum. Rarely, it can involve internal capsule, frontal lobe, and temporal lobe. This might lead to misdiagnosis as herpes encephalitis or demyelination as in our patient. MRI has a major role in early diagnosis of $L$. rhombencephalitis, as isolation of L. monocytogenes by culture or PCR may be possible only in up to 50 to $60 \%$ of cases. ${ }^{6}$

\section{Conflict of Interest}

None declared.

\section{References}

1 Arslan F, Ertan G, Emecen AN, Fillatre P, Mert A, Vahaboglu $\mathrm{H}$. Clinical presentation and cranial MRI findings of Listeria monocytogenes encephalitis: a literature review of case series. Neurologist 2018;23(6):198-203

2 Hatpoglu HG, Onbasioglu Gubuz M, Sakman B, Yuksel E. Diffusion weighted MRI in rhombenceephalits due to Listeria monocytogenes. Acta Radiol 2007;48:464

3 Reynaud L, Graf M, Gentile I, et al. A rare case of brainstem encephalitis by Listeria monocytogenes with isolated mesencephalic localization. Case report and review. Diagn Microbiol Infect Dis 2007;58(1):121-123

4 Salonga-Reyes A, Badve MS, Bhuta S, Broadley S, Jones A. Non-enhancing subcortical white matter lesions in central nervous system listeriosis. Intern Med J 2015;45(2):228-229

5 Mano T, Saito M, Yoshizawa T. Axonal invasion of Listeria monocytogenes: implications for early diagnosis with magnetic resonance imaging. J Neurol Sci 2017;373:7-8

6 Mansbridge CT, Grecu I, Li Voon Chong JS, Vandervelde C, Saeed K. Two cases of listeria rhombencephalitis. IDCases 2017; $11: 22-25$ 